\title{
Polynomial Generalizations of two-variable Ramanujan type identities
}

\author{
James Mc Laughlin \\ Department of Mathematics, 124 Anderson Hall, \\ West Chester University, West Chester, PA 19383 USA \\ jmclaughlin@wcupa.edu \\ Andrew V. Sills \\ Department of Mathematical Sciences, \\ 203 Georgia Avenue, Georgia Southern University, Statesboro, GA 30460 USA \\ asills@georgiasouthern.edu
}

Submitted: Jun 26, 2010; Accepted: Jun 14, 2011; Published: Jun 28, 2011

Mathematics Subject Classifications: 11B65, 05A10

Dedicated to Doron Zeilberger on the occasion of his sixtieth birthday.

"The progress of mathematics can be viewed as progress from the infinite to the finite." - Gian-Carlo Rota (1983)

\begin{abstract}
We provide finite analogs of a pair of two-variable $q$-series identities from Ramanujan's lost notebook and a companion identity.
\end{abstract}

\section{Introduction}

At the top of a page in the lost notebook [14, p. 33] (cf. [6, p. 99, Entry 5.3.1]):, Ramanujan recorded an identity equivalent to the following:

$$
\sum_{j=0}^{\infty} \frac{q^{2 j^{2}}\left(z q ; q^{2}\right)_{j}\left(q / z ; q^{2}\right)_{j}}{\left(q^{2} ; q^{2}\right)_{2 j}}=\frac{\left(z q^{3}, q^{3} / z, q^{6} ; q^{6}\right)_{\infty}}{\left(q^{2} ; q^{2}\right)_{\infty}}
$$

where we are employing the standard notation for rising $q$-factorials,

$$
(A ; q)_{\infty}:=(1-A)(1-A q)\left(1-A q^{2}\right) \cdots \text { and }(A ; q)_{n}:=\frac{(A ; q)_{\infty}}{\left(A q^{n} ; q\right)_{\infty}}
$$


and

$$
\left(A_{1}, A_{2}, \cdots, A_{r} ; q\right)_{\infty}:=\left(A_{1} ; q\right)_{\infty}\left(A_{2} ; q\right)_{\infty} \cdots\left(A_{r} ; q\right)_{\infty}
$$

In a recent paper [11], we found a partner to (1.1) that Ramanujan appears to have missed:

$$
\sum_{j=0}^{\infty} \frac{q^{j(j+1)}(z ; q)_{j}(q / z ; q)_{j+1}}{(q ; q)_{2 j+1}}=\frac{\left(z q^{2}, q / z, q^{3} ; q^{3}\right)_{\infty}}{(q ; q)_{\infty}} .
$$

Later on the same page of the lost notebook, Ramanujan recorded [6, p. 103, Entry $5.3 .5]$

$$
\sum_{j=0}^{\infty} \frac{q^{j^{2}}\left(z q ; q^{2}\right)_{j}\left(q / z ; q^{2}\right)_{j}}{\left(q ; q^{2}\right)_{j}\left(q^{4} ; q^{4}\right)_{j}}=\frac{\left(z q^{2}, q^{2} / z, q^{4} ; q^{4}\right)_{\infty}\left(-q ; q^{2}\right)_{\infty}}{\left(q^{2} ; q^{2}\right)_{\infty}} .
$$

For further discussion of these three identities, see [10].

Remark. Out of respect for Doron's ultra-finitist philosophy, we deliberately refrain from stating conditions on $q$ and $z$ which imply analytic convergence of the infinite series and products in (1.1)-(1.3).

The preceding identities stand out among identities of Rogers-Ramanujan type because they are two-variable series-product identities. While Rogers-Ramanujan type identities admit two-variable generalizations, most lose the infinite product representation in the two-variable case.

For example, in the standard two variable generalization of the first Rogers-Ramanujan identity,

$$
\sum_{j=0}^{\infty} \frac{z^{j} q^{j^{2}}}{(q ; q)_{j}}=\frac{1}{(z q ; q)_{\infty}} \sum_{n=0}^{\infty} \frac{(-1)^{j} z^{2 j} q^{j(5 j-1) / 2}\left(1-z q^{2 j}\right)(z ; q)_{j}}{(1-z)(q ; q)_{j}}
$$

the right hand side reduces to an infinite product only for certain particular values of $z$, e.g. $z=1$ gives the first Rogers-Ramanujan identity,

$$
\sum_{j=0}^{\infty} \frac{q^{j^{2}}}{(q ; q)_{j}}=\frac{1}{\left(q ; q^{5}\right)_{\infty}\left(q^{4} ; q^{5}\right)_{\infty}}
$$

while $z=q$ gives the second Rogers-Ramanujan identity,

$$
\sum_{j=0}^{\infty} \frac{q^{j(j+1)}}{(q ; q)_{j}}=\frac{1}{\left(q^{2} ; q^{5}\right)_{\infty}\left(q^{3} ; q^{5}\right)_{\infty}}
$$

after application of the Jacobi triple product identity [6, p. 17, Eq. (1.4.8)].

In $[16, \S 3]$, the second author presented nontrivial polynomial generalizations of all 130 Rogers-Ramanujan type identities appearing in Slater's paper [18]. All of Slater's identities involved one variable only. Here, we demonstrate that the methods employed in [16] can be used to obtain polynomial generalizations of the rarer species of two-variable $q$-series-product identities as well. 


\section{Polynomial Generalizations}

Define the standard binomial coëfficient by

$$
\left[\begin{array}{l}
A \\
B
\end{array}\right]_{q}:=\left\{\begin{array}{ll}
\frac{(q ; q)_{A}}{(q ; q)_{B}(q ; q)_{A-B}}, & \text { if } 0 \leq B \leq A \\
0, & \text { otherwise }
\end{array},\right.
$$

and the modified $q$-binomial coëfficient by

$$
\left[\begin{array}{l}
A \\
B
\end{array}\right]_{q}^{*}:=\left\{\begin{array}{ll}
1, & \text { if } A=-1 \text { and } B=0, \\
{\left[\begin{array}{l}
A \\
B
\end{array}\right]_{q},} & \text { otherwise }
\end{array} .\right.
$$

In [4], Andrews and Baxter define several $q$-analogs of trinomial coëfficients; we shall require one of them here:

$$
T_{0}(L, A ; q):=\sum_{r=0}^{L}(-1)^{r}\left[\begin{array}{l}
L \\
r
\end{array}\right]_{q^{2}}\left[\begin{array}{c}
2 L-2 r \\
L-A-r
\end{array}\right]_{q} .
$$

More recently, Andrews [3] introduced the following generalization of the $q$-binomial coëfficient:

$$
\left[\begin{array}{l}
A \\
B
\end{array} ; q, z\right]:= \begin{cases}0 & \text { if } B<0 \\
1 & \text { if } B=0 \text { or } B=A \\
\sum_{h=0}^{B} z^{h}\left[\begin{array}{c}
A-B+h-1 \\
h
\end{array}\right]_{q} & \text { if } 0<B<A \\
\left(z q^{A-B} ; q\right)_{B-A} & \text { if } B>A .\end{cases}
$$

The following polynomial generalizations of (1.4) are known:

$$
\sum_{j=0}^{n} z^{j} q^{j^{2}}\left[\begin{array}{l}
n \\
j
\end{array}\right]_{q}=\sum_{j=0}^{n}(-1)^{j} q^{j(5 j-1) / 2}\left(1-z q^{2 j}\right)\left[\begin{array}{c}
n \\
j
\end{array}\right]_{q} \frac{1}{\left(z q^{j} ; q\right)_{n+1}}
$$

$($ see $[1,7,12,21])$,

$$
\begin{aligned}
\sum_{j=0}^{n} z^{n} q^{n^{2}}=\sum_{0 \leq 2 j \leq n}(-1)^{j} z^{2 j} q^{j(5 j-1) / 2}\left(1-z q^{2 j}\right)\left[\begin{array}{c}
n \\
j
\end{array}\right]_{q}\left[\begin{array}{c}
n-j \\
j
\end{array}\right]_{q}(q ; q)_{j} & \\
& \times \frac{\left(z^{2} q^{n+2 j+1} ; q\right)_{n-2 j}}{\left(z q^{j} ; q\right)_{n-j+1}}
\end{aligned}
$$

$[8, \mathrm{Eq} \cdot(3.5)]$, and

$$
\begin{aligned}
\sum_{j=0}^{n} z^{j} q^{j^{2}}\left[\begin{array}{c}
n \\
j
\end{array} ; q, q\right]= & \sum_{0 \leq 2 j \leq n}(-1)^{j} z^{2 j} q^{j(5 j-1) / 2}\left[\begin{array}{c}
n \\
j
\end{array} ; q, q\right]\left[\begin{array}{c}
2 n+1-2 j \\
n-2 j
\end{array} ;, z q^{j}\right] \\
& -\sum_{0 \leq 2 j \leq n-1}(-1)^{j} z^{2 j+1} q^{j(5 j+3) / 2}\left[\begin{array}{c}
n \\
j
\end{array} ; q, q\right]\left[\begin{array}{c}
2 n-2 j \\
n-2 j-1
\end{array} ; q, z q^{j}\right]
\end{aligned}
$$

[3, p. 41, Eq. (1.11)]. 
Andrews [3] notes that one of his motivations for introducing (2.3) is that both sides of the equation are clearly polynomials term by term, whereas this is not the case for the right hand sides of (2.1) and (2.2). The polynomial identities we introduce below also have this desirable feature.

Notice that in each of the identities below, the summands have finite support, and follow the natural bounds (i.e. each summation could be taken over all integers, and no nonzero terms would be added).

Identity 2.1 (Polynomial Generalization of (1.1)).

$$
\begin{aligned}
\sum_{j=0}^{\lfloor n / 2\rfloor} \sum_{h=0}^{j} \sum_{i=0}^{j}(-1)^{h+i} z^{h-i} q^{h^{2}+i^{2}+2 j^{2}}\left[\begin{array}{l}
j \\
h
\end{array}\right]_{q^{2}} & {\left[\begin{array}{l}
j \\
i
\end{array}\right]_{q^{2}}\left[\begin{array}{c}
j+\left\lfloor\frac{n-h-i}{2}\right\rfloor \\
2 j
\end{array}\right]_{q^{2}} } \\
& =\sum_{j=-\infty}^{\infty}(-1)^{j} z^{j} q^{3 j^{2}}\left[\begin{array}{c}
n-1 \\
\left\lfloor\frac{n+3 j-1}{2}\right\rfloor
\end{array}\right]_{q^{2}}+\epsilon_{n}(z, q),
\end{aligned}
$$

where

$$
\epsilon_{n}(z, q)= \begin{cases}\sum_{j=-\infty}^{\infty} z^{2 j} q^{12 j^{2}+6 j+n}\left[\begin{array}{c}
n-1 \\
\frac{n+6 j}{2}
\end{array}\right]_{q^{2}}^{*} & \text { if } 2 \mid n \\
-\sum_{j=-\infty}^{\infty} z^{2 j-1} q^{12 j^{2}-6 j+n}\left[\begin{array}{c}
n-1 \\
\frac{n+6 j-3}{2}
\end{array}\right]_{q^{2}}^{*} & \text { if } 2 \nmid n\end{cases}
$$

Identity 2.2 (Polynomial Generalization of (1.2)).

$$
\begin{aligned}
& \sum_{j=0}^{\lfloor n / 2\rfloor} \sum_{h=0}^{j} \sum_{i=0}^{j}(-1)^{h+i} z^{h-i} q^{\left(\begin{array}{c}
h \\
2
\end{array}\right)+\left(\begin{array}{l}
i \\
2
\end{array}\right)+j(j+1)}\left[\begin{array}{l}
j \\
h
\end{array}\right]_{q}\left[\begin{array}{c}
j+1 \\
i
\end{array}\right]_{q}\left[\begin{array}{c}
j+1+\left\lfloor\frac{n-h-i}{2}\right\rfloor \\
2 j+1
\end{array}\right]_{q} \\
& =\sum_{j=-\infty}^{\infty}(-1)^{j} z^{j} q^{j(3 j+1) / 2}\left[\begin{array}{c}
n \\
\left\lfloor\frac{n+3 j+2}{2}\right\rfloor
\end{array}\right]_{q}+\epsilon_{n}(z, q) \text {, }
\end{aligned}
$$

where

$$
\epsilon_{n}(z, q)= \begin{cases}\sum_{j=-\infty}^{\infty} z^{2 j} q^{6 j^{2}-2 j+n / 2}\left[\begin{array}{c}
n \\
\frac{n}{2}+3 j
\end{array}\right]_{q} & \text { if } 2 \mid n, \\
-\sum_{j=-\infty}^{\infty} z^{2 j+1} q^{6 j^{2}+4 j+\frac{1}{2}+\frac{n}{2}}\left[\begin{array}{c}
n \\
\left.\frac{n+6 j+3}{2}\right]_{q}
\end{array}\right. & \text { if } 2 \nmid n .\end{cases}
$$


Identity 2.3 (Polynomial Generalization of (1.3)).

$$
\begin{aligned}
\sum_{j=0}^{\lfloor n / 2\rfloor} \sum_{h=0}^{j} \sum_{i=0}^{j} \sum_{\ell=0}^{n-h-i}(-1)^{h+i+\ell} z^{h-i} q^{h^{2}+i^{2}+j^{2}+2 \ell}\left[\begin{array}{l}
j \\
h
\end{array}\right]_{q^{2}}\left[\begin{array}{l}
j \\
i
\end{array}\right]_{q^{2}}\left[\begin{array}{c}
j+\ell-1 \\
\ell
\end{array}\right]_{q^{2}}^{*} \\
\times\left[\begin{array}{c}
n-h-i+j-\ell \\
2 j
\end{array}\right]_{q} \\
=\sum_{j=-\infty}^{\infty}(-1)^{j} z^{j} q^{2 j^{2}}\left(T_{0}(n, 2 j ; q)+T_{0}(n-1,2 j ; q)\right)
\end{aligned}
$$

\section{Derivation and a method of proof}

\section{$3.1 \quad$ Identity 2.1}

Recall the following consequences of the $q$-binomial theorem:

$$
\begin{gathered}
(t ; q)_{j}=\sum_{h=0}^{j}(-1)^{h} t^{h} q^{h(h-1) / 2}\left[\begin{array}{l}
j \\
h
\end{array}\right]_{q} \\
\frac{1}{(t ; q)_{j}}=\sum_{h=0}^{\infty} t^{h}\left[\begin{array}{c}
h+j-1 \\
h
\end{array}\right]_{q}^{*}
\end{gathered}
$$

The derivation of Identity 2.1 is via the method used for the derivations of polynomial versions of Rogers-Ramanujan type identities (in $q$ only) as introduced by Andrews [2, Chapter 9], and further explored by Santos [15] and the second author [16, 17]. We shall consider the details of (1.1) only; (1.2) and (1.3) may be treated analogously.

We begin with the left hand side of (1.1)

$$
\phi(z, q):=\sum_{j=0}^{\infty} \frac{q^{2 j^{2}}\left(z q ; q^{2}\right)_{j}\left(q / z ; q^{2}\right)_{j}}{\left(q^{2} ; q^{2}\right)_{2 j}} .
$$

Now define the following generalization of $\phi(z, q)$ :

$$
f(t):=f(t ; z, q):=\sum_{j=0}^{\infty} \frac{t^{2 j}(1+t) q^{2 j^{2}}\left(t z q ; q^{2}\right)_{j}\left(t q / z ; q^{2}\right)_{j}}{\left(t^{2} ; q^{2}\right)_{2 j+1}}
$$

and let $P_{n}(z, q)$ be defined by

$$
f(t)=\sum_{n=0}^{\infty} P_{n}(z, q) t^{n}
$$

Note that

$$
\lim _{t \rightarrow 1-}(1-t) f(t ; z, q)=\phi(z, q)
$$


and

$$
\begin{gathered}
\lim _{n \rightarrow \infty} P_{n}(z, q)=\phi(z, q) . \\
f(t)=\sum_{j=0}^{\infty} \frac{t^{2 j}(1+t) q^{2 j^{2}}\left(t z q ; q^{2}\right)_{j}\left(t q / z ; q^{2}\right)_{j}}{\left(t^{2} ; q^{2}\right)_{2 j+1}} \\
=\frac{1}{1-t}+\sum_{j=1}^{\infty} \frac{t^{2 j}(1+t) q^{2 j^{2}}\left(t z q ; q^{2}\right)_{j}\left(t q / z ; q^{2}\right)_{j}}{\left(t^{2} ; q^{2}\right)_{2 j+1}} \\
=\frac{1}{1-t}+\sum_{j=0}^{\infty} \frac{t^{2 j+2} q^{2 j^{2}+4 j+2}\left(t z q ; q^{2}\right)_{j+1}\left(t q / z ; q^{2}\right)_{j+1}}{\left(t^{2} ; q^{2}\right)_{2 j+3}} \\
=\frac{1}{1-t}+\frac{t^{2} q^{2}(1-t z q)(1-t q / z)}{\left(1-t^{2} q^{2}\right)\left(1+t q^{2}\right)(1-t)} f\left(t q^{2}\right) .
\end{gathered}
$$

Thus,

$$
\left(1-t^{2} q^{2}\right)\left(1+t q^{2}\right)(1-t) f(t)=\left(1-t^{2} q^{2}\right)\left(1+t q^{2}\right)+t^{2} q^{2}(1-t z q)(1-t q / z) f\left(t q^{2}\right),
$$

which immediately implies

$$
\begin{aligned}
f(t)=\left(1+t q^{2}-t^{2} q^{2}-t^{3} q^{4}\right)+\left(\left(1-q^{2}\right) t\right. & \left.+2 q^{2} t^{2}+\left(q^{4}-q^{2}\right) t^{3}-q^{4} t^{4}\right) f(t) \\
& +\left(q^{2} t^{2}-\left(z+z^{-1}\right) q^{3} t^{3}+q^{4} t^{4}\right) f\left(t q^{2}\right) .
\end{aligned}
$$

Upon recalling that $f(t)=\sum_{n=0}^{\infty} P_{n}(z, q) t^{n}$, and extracting the coëfficients of $t^{n}$ from (3.5), we find that the $P_{n}=P_{n}(z, q)$ satisfy the fourth order recurrence

$$
\begin{aligned}
P_{n}=\left(1-q^{2}\right) P_{n-1}+\left(2 q^{2}+q^{2 n-2}\right) P_{n-2}+\left(q^{4}-q^{2}-\left(z+z^{-1}\right) q^{2 n-3}\right) P_{n-3} \\
+\left(q^{2 n-4}-q^{4}\right) P_{n-4}
\end{aligned}
$$

with initial conditions

$$
P_{0}=P_{1}=1 ; \quad P_{2}=1+q^{2} ; \quad P_{3}=1+q^{2}-\left(z+z^{-1}\right) q^{3} .
$$

Thus we now have a full characterization of the $P_{n}(z, q)$ via a recurrence with initial conditions. 
Next, we use $f(t)$ to derive the left hand side of Identity 2.1.

$$
\begin{aligned}
& \sum_{n=0}^{\infty} P_{n}(z, q) t^{n}=f(t) \\
& =\sum_{j=0}^{\infty} \frac{t^{2 j}(1+t) q^{2 j^{2}}\left(t z q ; q^{2}\right)_{j}\left(t q / z ; q^{2}\right)_{j}}{\left(t^{2} ; q^{2}\right)_{2 j+1}} \\
& =\sum_{j=0}^{\infty} t^{2 j}(1+t) q^{2 j^{2}} \sum_{h=0}^{j}(-t z q)^{h} q^{h^{2}-h}\left[\begin{array}{l}
j \\
h
\end{array}\right]_{q^{2}} \sum_{i=0}^{j}\left(-t q z^{-1}\right)^{i} q^{i^{2}-i}\left[\begin{array}{l}
j \\
i
\end{array}\right]_{q^{2}} \\
& \times \sum_{r=0}^{\infty} t^{2 r}\left[\begin{array}{c}
2 j+r \\
r
\end{array}\right]_{q^{2}}(\text { by }(3.1) \text { and }(3.2)) \\
& =\sum_{h, i, j, r \geq 0} t^{2 j+h+i}\left(t^{2 r}+t^{2 r+1}\right)(-1)^{h+i} q^{2 j^{2}+h^{2}+i^{2}} z^{h-i} \\
& \times\left[\begin{array}{l}
j \\
h
\end{array}\right]_{q^{2}}\left[\begin{array}{l}
j \\
i
\end{array}\right]_{q^{2}}\left[\begin{array}{c}
2 j+r \\
2 j
\end{array}\right]_{q^{2}} \\
& =\sum_{h, i, j, r \geq 0} t^{2 j+h+i+s}(-1)^{h+i} q^{2 j^{2}+h^{2}+i^{2}} z^{h-i} \\
& \times\left[\begin{array}{l}
j \\
h
\end{array}\right]_{q^{2}}\left[\begin{array}{l}
j \\
i
\end{array}\right]_{q^{2}}\left[\begin{array}{c}
\left\lfloor\frac{s}{2}\right\rfloor+2 j \\
2 j
\end{array}\right]_{q^{2}}(\text { where } s=2 r \text { or } s=2 r+1) \\
& =\sum_{n=0}^{\infty} t^{n} \sum_{h, i, j \geq 0}(-1)^{h+i} q^{2 j^{2}+h^{2}+i^{2}} z^{h-i}\left[\begin{array}{l}
j \\
h
\end{array}\right]_{q^{2}}\left[\begin{array}{l}
j \\
i
\end{array}\right]_{q^{2}}\left[\begin{array}{c}
j+\left\lfloor\frac{n-h-i}{2}\right\rfloor \\
2 j
\end{array}\right]_{q^{2}} \\
& \text { (where } n=2 j+h+i+s \text { ). }
\end{aligned}
$$

Compare coëfficients of $t^{n}$ in the extremes to find

$$
P_{n}(z, q)=\sum_{h, i, j \geq 0}(-1)^{h+i} z^{h-i} q^{2 j^{2}+h^{2}+i^{2}}\left[\begin{array}{l}
j \\
h
\end{array}\right]_{q^{2}}\left[\begin{array}{l}
j \\
i
\end{array}\right]_{q^{2}}\left[\begin{array}{c}
j+\left\lfloor\frac{n-h-i}{2}\right\rfloor \\
2 j
\end{array}\right]_{q^{2}} .
$$

Next, after some inspired guesswork, (see $[5,16,17]$ for details) we define the polynomials

$$
\begin{aligned}
& Q_{n}=Q_{n}(z, q) \\
& := \begin{cases}\sum_{k} z^{2 k} q^{12 k^{2}}\left[\begin{array}{c}
2 m \\
m+3 k
\end{array}\right]_{q^{2}}-z^{-2 k-1} q^{12 k^{2}+12 k+3}\left[\begin{array}{c}
2 m-1 \\
m+3 k+1
\end{array}\right]_{q^{2}}, & \text { if } n=2 m \\
\sum_{k} z^{2 k} q^{12 k^{2}}\left[\begin{array}{c}
2 m \\
m+3 k
\end{array}\right]_{q^{2}}-z^{-2 k-1} q^{12 k^{2}+12 k+3\left[\begin{array}{c}
2 m+1 \\
m+3 k+2
\end{array}\right]_{q^{2}},} & \text { if } n=2 m+1\end{cases} \\
& =\sum_{j=-\infty}^{\infty}(-1)^{j} z^{j} q^{3 j^{2}}\left[\begin{array}{c}
n-1 \\
\left\lfloor\frac{n+3 j-1}{2}\right\rfloor
\end{array}\right]_{q^{2}}+\epsilon_{n}(z, q),
\end{aligned}
$$


where

$$
\epsilon_{n}(z, q)=\left\{\begin{array}{ll}
\sum_{j=-\infty}^{\infty} z^{2 j} q^{12 j^{2}+6 j+n}\left[\begin{array}{c}
n-1 \\
\frac{n+6 j}{2}
\end{array}\right]_{q^{2}}^{*} & \text { if } 2 \mid n \\
-\sum_{j=-\infty}^{\infty} z^{2 j-1} q^{12 j^{2}-6 j+n}\left[\begin{array}{c}
n-1 \\
\frac{n+6 j-3}{2}
\end{array}\right]_{q^{2}}^{*} & \text { if } 2 \nmid n
\end{array} .\right.
$$

Our goal is to show that the $P_{n}(z, q)$ and $Q_{n}(z, q)$ are in fact one and the same, thus giving us Identity 2.1. We would like to use a computer implementation of the $q$-Zeilberger algorithm $[13,19,20,21,22]$ to simply show that the $Q_{n}$ satisfy the recurrence (3.6), and then upon checking that the $Q_{n}$ satisfy the initial conditions (3.7), we would be done. Unfortunately, the implementations of the $q$-Zeilberger algorithm currently available do not allow for direct input of summands as complex as those under consideration here. And the corresponding certificate function would likely be rather horrendous. Further, it is unlikely that the $q$-Zeilberger algorithm would produce a minimal recurrence for the $Q_{n}$. So, the traditional automated proof would require a certain amount of pre-processing and post-processing.

The referee pointed out that the proof can be completed by showing that the coëfficients of $z^{j}$ on both sides agree for all $j$, which boils down to proving certain recurrences for $q$-binomial coëfficients, i.e. look at the coëfficient of $z^{j}$ in the recurrence (3.6), and show that $Q_{n}$ satisfies the same recurrence. The recurrence depends on the parity of $n$ and $j$. So, e.g., for the case where $n$ and $j$ are both even, let $n=2 m$ and $j=2 k$; then show that

$$
\begin{aligned}
& {\left[\begin{array}{c}
2 m \\
m+3 k
\end{array}\right]_{q}=\left[\begin{array}{c}
2 m-2 \\
m+3 k-1
\end{array}\right]_{q}\left(1+q+q^{2 m+1}\right)-\left[\begin{array}{c}
2 m-3 \\
m+3 k
\end{array}\right]_{q} q^{6 k+2 m}} \\
& -\left[\begin{array}{c}
2 m-3 \\
m+3 k-3
\end{array}\right]_{q} q^{2 m-6 k}+\left[\begin{array}{c}
2 m-4 \\
m+3 k-2
\end{array}\right]_{q}\left(q-q^{2 m-2}\right) \text {. }
\end{aligned}
$$

This is a straightfoward (albeit tedious) exercise and precisely the type of verification that can be carried out with the aid of the RRtools Maple package; see [17] for further discussion.

\section{$3.2 \quad$ Identity 2.2}

The derivation is analogous to that of Identity 2.1. The analogous " $t$-generalization" of the sum side of (1.2) is

$$
f(t ; z, q)=\sum_{j=0}^{\infty} \frac{t^{2 j} q^{j^{2}+j}(1+t)(t z ; q)_{j}(t q / z ; q)_{j+1}}{\left(t^{2} ; q\right)_{2 j+1}}=\sum_{n=0}^{\infty} P_{n}(z, q) t^{n} .
$$

The recurrence and initial conditions satisfied by this new $P_{n}=P_{n}(z, q)$ are

$$
\begin{aligned}
P_{n}=(1-q) P_{n-1}+\left(2 q+q^{n}\right) P_{n-2}+\left(q^{2}-q-\left(z q^{2}+z^{-1} q^{3}\right) q^{n-3}\right) & P_{n-3} \\
& +\left(q^{n-1}-q^{2}\right) P_{n-4}
\end{aligned}
$$


with

$$
\begin{aligned}
P_{0}=1, \quad P_{1}=1-q / z, \quad P_{2}=1+\left(1-z^{-1}\right) q+q^{2}, \\
P_{3}=1+\left(1-z^{-1}\right) q+\left(1-z-z^{-1}\right) q^{2}-q^{3} / z-q^{4} / z .
\end{aligned}
$$

Again, upon verifying that the coëfficient of $z^{j}$ in the recurrence (3.9) satisfies the same recurrence as the right hand side of Identity 2.2 , the proof is complete.

\section{$3.3 \quad$ Identity 2.3}

The $t$-generalization of the sum side of (1.3) is

$$
f(t ; z, q)=\sum_{j=0}^{\infty} \frac{t^{j} q^{j^{2}}(1+t)\left(t z q ; q^{2}\right)_{j}\left(t q / z ; q^{2}\right)_{j}}{\left(t q ; q^{2}\right)_{j}\left(t^{2} ; q^{4}\right)_{j+1}}=\sum_{n=0}^{\infty} P_{n}(z, q) t^{n}
$$

The recurrence and initial conditions for this third $P_{n}=P_{n}(z, q)$ are

$$
P_{n}=\left(1+q-q^{2}+q^{2 n-1}\right) P_{n-1}+\left(q^{3}+q^{2}-q-\left(z+z^{-1}\right) q^{2 n-2}\right) P_{n-2}+\left(q^{2 n-3}-q^{3}\right) P_{n-3}
$$

with

$$
P_{0}=1, \quad P_{1}=1+q, \quad P_{2}=1+q+\left(1-z-z^{-1}\right) q^{2}+q^{4} .
$$

To complete this proof, we note that unlike Identities 2.1 and 2.2 , the coëfficient of $z^{j}$ in Identity 2.3 does not depend on the parity of $n$ or $j$. However, as the $q$-trinomial coëfficients are involved, the required recurrence verification, while analogous to that of the two previous identities where only $q$-binomial coëfficients were involved, is somewhat more complicated in detail; see [5, 17]. Nonetheless, this verification has been carried out, thus completing the proof.

\section{Challenge}

We leave it as a challenge to produce fully automated proofs for Identities 2.1-2.3.

\section{Acknowledgments}

Many thanks to Doron Zeilberger for revolutionizing the way we approach the discovery and proof of identities, especially those of the hypergeometric and $q$-hypergeometric type.

We also thank the anonymous referee for catching a number of typographical errors and pointing out some details that needed clarification.

\section{References}

[1] G. E. Andrews, Problem 74-12, SIAM Review 16 (1974). 
[2] G. E. Andrews, q-Series: Their Development and Application in Analysis, Number Theory, Combinatorics, Physics and Computer Algebra, C.B.M.S. Regional Conference Series in Math, No. 66, American Math. Soc. Providence, 1986.

[3] G. E. Andrews, a-Gaussian polynomials and finite Rogers-Ramanujan identities, in: Theory and Applications of Special Functions: a Volume Dedicated to Mizan Rahman, M. Ismail and E. Koelink, eds., 39-60. Springer, New York, 2005.

[4] G. E. Andrews and R. J. Baxter, Lattice gas generalization of the hard hexagon model III: $q$-trinomial coefficients, J. Statist. Phys 47 (1987) 298-330.

[5] G. E. Andrews and R. J. Baxter, Scratchpad explorations for elliptic theta functions, In: D. V. Chudnovsky and R. D. Jenks (eds.), Computers in Mathematics. Lecture Notes in Pure and Applied Mathematics, vol. 125, pp. 17-33, Marcel Dekker, 1990.

[6] G. E. Andrews and B. C. Berndt, Ramanujan's Lost Notebook, Part II, Springer, 2009.

[7] D. M. Bressoud, Solution to problem 74-12, SIAM Review 23 (1981) 101-104.

[8] D. M. Bressoud, Some identities for terminating $q$-series, Math Proc. Cambridge Phil. Soc. 89 (1981) 211-223.

[9] S. B. Ekhad and S. Tre, A purely verification proof of the first Rogers-Ramanujan identity, J. Combin. Theory Ser. A 54(2) (1990) 309-311.

[10] J. McLaughlin and A. V. Sills, On a pair of identities from Ramanujan's lost notebook, preprint, 2010.

[11] J. McLaughlin, A. V. Sills and P. Zimmer, Some implications of Chu's ${ }_{10} \psi_{10}$ extension of Bailey's ${ }_{6} \psi_{6}$ summation formula, preprint, 2009.

[12] P. Paule, Short and easy computer proofs of the Rogers-Ramanujan identities and identities of similar type, Electronic J. Combin. 1 (1994), \#R10, 9 pp.

[13] M. Petkovšek, H. S. Wilf and D. Zeilberger, $A=B$. A. K. Peters, Wellesley, MA, 1996.

[14] S. Ramanujan, The Lost Notebook and Other Unpublished Papers, Narosa, 1987.

[15] J. P. O. Santos, Computer algebra and identities of the Rogers-Ramanujan type, Ph.D. thesis, Pennsylvania State University, 1991.

[16] A. V. Sills, Finite Rogers-Ramanujan type identities, Electronic J. Combin. 10(1) (2003) \#R13, 122 pp.

[17] A. V. Sills, RRtools-a Maple package for aiding the discovery and proof of finite Rogers-Ramanujan type identities, J. Symbolic Comput. 37 (2004) 415-448.

[18] L. J. Slater, Further identities of the Rogers-Ramanujan type, Proc. London Math. Soc. (2) 54 (1952) 147-167.

[19] H. S. Wilf and D. Zeilberger, Rational functions certify combinatorial identities, J. Amer. Math. Soc. 3 (1990), 147-158.

[20] H. S. Wilf and D. Zeilberger, Rational function certification of hypergeometric multiintegral/sum/"q" identities, Invent. Math. 108 (1992) 575-633.

[21] D. Zeilberger, A fast algorithm for proving terminating hypergeometric identities, Discrete Math. 80 (1990) 207-211.

[22] D. Zeilberger, The method of creative telescoping, J. Symbolic Comput. 11 (1991) 195-204. 